\title{
Dispersion in DLA metallicities and deuterium abundances
}

\section{Irina Dvorkin ${ }^{1}$, Joseph Silk ${ }^{1,2,3,4}$, Elisabeth Vangioni ${ }^{1}$, Patrick Petitjean $^{1}$ and Keith A. Olive ${ }^{5}$}

${ }^{1}$ Sorbonne Universités, UPMC Univ Paris 6 et CNRS, UMR 7095, Institut dAstrophysique de Paris, 98 bis bd Arago, F-75014 Paris, France

${ }^{2}$ AIM-Paris-Saclay, CEA/DSM/IRFU, CNRS, Univ. Paris VII, F-91191 Gif-sur-Yvette, France

${ }^{3}$ Department of Physics and Astronomy, The Johns Hopkins University, Baltimore, MD 21218, USA

${ }^{4}$ BIPAC, University of Oxford, 1 Keble Road, Oxford OX1 3RH, UK

${ }^{5}$ William I. Fine Theoretical Physics Institute, School of Physics and Astronomy, University of Minnesota, Minneapolis, MN 55455, USA

\begin{abstract}
Recent chemical abundance measurements of damped Lyman-alpha absorbers (DLAs) revealed a large intrinsic scatter in their metallicities. We discuss a semi-analytic model that was specifically designed to study this scatter by tracing the chemical evolution of the interstellar matter in small regions of the Universe with different mean density, from over- to underdense regions. It is shown that different histories of structure formation in these regions are reflected in the chemical properties of the proto-galaxies. We also address deuterium abundance measurements, which constitute a complementary probe of the star formation and infall histories.
\end{abstract}

Keywords. ISM: abundances, galaxies: abundances, galaxies: evolution, galaxies: ISM

\section{Introduction}

Numerous studies of damped Ly $\alpha$ absorbers (DLAs) conducted over the past several years have provided extensive samples of high-redshift systems (e.g. Prochaska et al. 2005). High resolution observations of nearly 250 of these systems established a statistically significant decrease of DLA metallicity with increasing redshift (e.g. Rafelski et al. 2012) and a large intrinsic dispersion of $\sim 0.5$ dex out to $z \sim 5$. We study this dispersion in the context of cosmological structure formation with an efficient semi-analytic model and explore the evolution of DLA metallicities and deuterium abundances.

\section{Cosmological chemical evolution model}

We take $V_{\text {tot }}=10^{6}(\mathrm{Mpc} / \mathrm{h})^{3}$ as the total comoving volume in our calculation and divide it into 1000 smaller regions $\Delta V_{i}=10^{3}(\mathrm{Mpc} / \mathrm{h})^{3}$. The volume $V_{\text {tot }}$ is populated with dark matter (DM) halos according to the Sheth-Tormen mass function (MF) at $z=0$ accounting for large-scale clustering effects: regions with large-scale overdensities form halos more easily. We use the results of Barkana \& Loeb (2004), for the MF bias caused by clustering. We then build a merger tree for each halo using the algorithm in the GALFORM model (Parkinson et al. 2008) and follow its evolution backwards in time up to $z_{f}=15$. We use $M_{\text {min }}=10^{8} M_{\odot}$ as the minimal halo mass able to form stars. Our next step is to calculate the mean mass fraction in collapsed structures, $f_{\text {coll }}$ and the mean escape velocity in each region $i$. Those regions that host a group or a cluster at low redshift have a higher concentration of structures already present at higher redshift, whereas present day voids are relatively empty at early times. This small-scale 


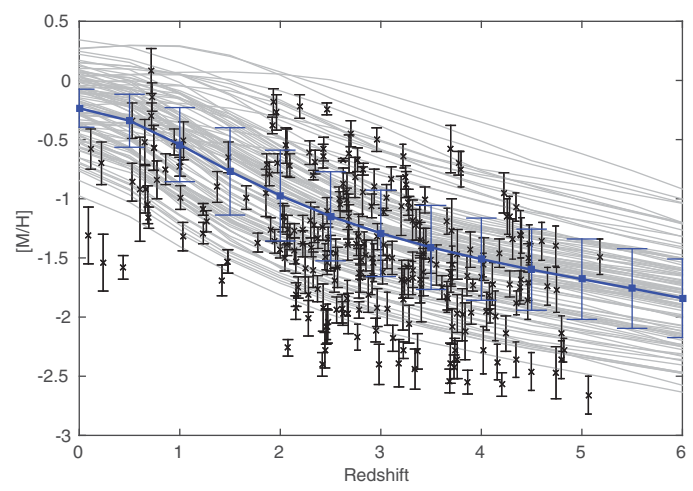

Figure 1. Metallicity abundance evolution in 100 regions, each with a volume of $\Delta V_{i}=10^{3}$ $\mathrm{Mpc}^{3} / \mathrm{h}^{3}$ (thin grey lines) and their mean (thick blue line) for $M_{\min }=10^{8} M_{\odot} / \mathrm{h}$. The dispersion among the realizations and their mean is represented by the blue points with large error bars. Black crosses represent data from Rafelski et al. (2012).

inhomogeneity creates the observed dispersion in the different observables. The resulting $f_{\text {coll }}$ and escape velocity for each region serve as inputs for the chemical evolution code developed in Daigne et al. (2004; 2006), which follows the exchange of mass between the gas within and outside of collapsed structures, the star formation rate (SFR) at each redshift and the rate of metal production in stars. Our model is calibrated so as to match the cosmic SFR from Behroozi et al. (2013). Further details on our model can be found in Dvorkin et al. $(2015 ; 2016)$.

\section{Results}

In Figure 1 we show the evolution of the metal abundance and compare it to observations of DLAs from Rafeslki et al. (2012). The results shown here include an estimate of the contribution from the mass-metallicity relation using the dispersion in stellar masses within each region (see Dvorkin et al. 2015 for details). It can be seen that our model successfully reproduces the observed dispersion with the exception of very metal-poor systems.

Deuterium is created during big bang nucleosynthesis (BBN) and is destroyed in stars. Since deuterium is not created after BBN its abundace constitutes a sensitive probe of the star formation and gas infall history of any given galaxy. The mean evolution of deuterium abundance in our model assuming the primordial value of $10^{5}(\mathrm{D} / \mathrm{H})_{p}=2.45$ (Coc et al. 2015) is shown by the thick black line in Figure 2. The thin grey lines show the evolution in 100 individual regions in our model. It can be seen that even in the most extreme cases the deuterium abundance reduces to about $\sim 1 / 3$ of its primordial value, reflecting the overall inefficiency of star formation. We also find that in some cases the evolution of deuterium abundance is non-monotonic due the complex interplay between star formation, which depletes deuterium, and infall of primordial gas which raises its abundance (see Dvorkin et al. 2016).

\section{Discussion}

We have shown that the dispersion in the fraction of collapsed structures, escape velocity and SFR between different regions in the Universe contributes to the dispersion in the metallicity-redshift relation of the DLAs. In addition, we calculated the disperison 


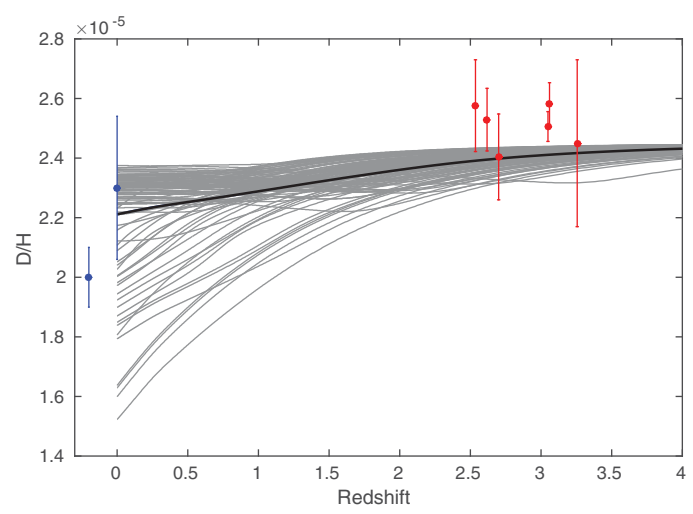

Figure 2. The evolution of deuterium abundance in each region (thin grey lines) and the mean (thick black line). Measurements of $z \sim 3$ DLAs from Cooke et al. (2014) and Riemer-Sørensen et al. (2015), local ISM measurements from Linsky et al. (2006) and Prodanović et al. (2010) (one of the points at $z=0$ is shifted for clarity).

in deuterium abundances expected from different structure formation histories. More observational and theoretical work is needed to improve our understanding of the relation between deuterium and metal abundances, which can provide important constraints on galaxy formation models.

\section{Acknowledgements}

Irina Dvorkin thanks the organizers for an interesting and stimulating conference. The work of ID and JS was supported by the ERC Project No. 267117 (DARK) hosted by Université Pierre et Marie Curie (UPMC) - Paris 6, PI J. Silk. JS acknowledges the support of the JHU by NSF grant OIA-1124403. The work of KAO was supported in part by DOE grant DE-SC0011842 at the University of Minnesota. This work has been carried out at the ILP LABEX (under reference ANR-10-LABX-63) supported by French state funds managed by the ANR within the Investissements d'Avenir programme under reference ANR-11-IDEX-0004-02.

\section{References}

Barkana, R. \& Loeb, A., 2004, ApJ, 609, 474

Behroozi, P. S., Wechsler, R. H. \& Conroy, C., 2013, ApJ, 770, 57

Coc, A., Petitjean, P., Uzan, J.-P., Vangioni, E., Descouvemont, P., Illiadis, C., \& Longland, R., 2015, Phys. Rev. D, 92, 12

Cooke, R. J., Pettini, M., Jorgenson, R. A., Murphy, M. T., \& Steidel, C. C., 2014, ApJ, 781, 31

Daigne, F., Olive, K. A., Vangioni-Flam E., Silk, J., \& Audouze, J., 2004, ApJ, 617, 693

Daigne, F., Olive, K. A., Silk, J., Stoehr, F., \& Vangioni, E., 2006, ApJ, 647, 773

Dvorkin, I., Silk, J., Vangioni, E., Petitjean, P., \& Olive, K. A., 2015, MNRAS, 452, L36

Dvorkin, I., Vangioni, E., Silk, J., Petitjean, P., \& Olive, K. A., 2016, MNRAS, 458, L104

Linsky, J. L. et al., 2006, ApJ, 647, 1106

Parkinson, H., Cole, S., \& Helly, J., 2008, MNRAS, 383, 557

Prochaska, J. X., Herbert-Fort S., \& Wolfe, A. M., 2005, ApJ, 635, 123

Prodanović T., Steigman, G., \& Fields, B. D., 2010, MNRAS, 406, 1108

Rafelski, M., Wolfe, A. M., Prochaska, J. X., Neeleman, M., \& Mendez, A. J., 2012, ApJ, 755, 89

Riemer-Sørensen S. et al., 2015, MNRAS, 447, 2925 